\title{
Integrated Guidance and Control Design For Time-Constrained Interception
}

\author{
Abhinav Sinha ${ }^{\dagger}$, Shashi Ranjan Kumar ${ }^{\circledR}$ and Dwaipayan Mukherjee ${ }^{\odot *}$
}

\begin{abstract}
This paper proposes integrated guidance and control design to intercept a non-maneuvering target at a pre-specified time of interception. The problem is addressed considering nonlinear engagement kinematics and the interceptor is steered using the combined effects of canard as well as tail configurations (dual control interceptors). Different formulations of time-to-go, without the restrictive assumption of interceptor's small heading angle, have been used in deriving the guidance commands, allowing the proposed strategies to remain effective over a wide range of impact time values. A weighted effort allocation scheme, in canard and tail deflections, has been proposed to generate the required lateral acceleration. The overall design uses sliding mode control owing to its simplicity of design. Finally, simulations are presented for various scenarios, including impaired actuator, vindicating the efficacy of the proposed technique.
\end{abstract}

Keywords - Integrated guidance and control, impact time, sliding mode control, nonlinear guidance, deviated pursuit .

\section{Introduction}

In recent years, constrained terminal phase guidance has received a lot of attention, especially in improving endgame performance. One of such constraints is the requirement of simultaneous arrival, also known as salvo [1], for which, control over impact time is necessary. The customary approach treats guidance and control design as two separate processes. By only considering kinematics of the engagement, the guidance system generates suitable commands to be tracked by the control system, whose response is assumed ideal or similar to that of a low pass filter [2]. Due to unexpected flight conditions, one cannot expect the response of the control system to be ideal. Disregarding the effect of controller dynamics while designing the guidance command can lead to incongruities between actual and ideal performances of the control system, engendering unsatisfactory behavior, especially in the endgame [3,4]. However, integrating separate guidance and control loops into a single loop, to directly generate the fin deflections, significantly enhances the endgame performance of the homing interceptor [4-6].

\footnotetext{
${ }^{* \dagger}$ Corresponding author.
}

A. Sinha and S.R. Kumar are with the Intelligent Systems \& Control Lab, Department of Aerospace Engineering, Indian Institute of Technology Bombay, Powai- 400076, Mumbai, India. e-mails: \{abhinavsinha,srk\}@aero.iitb.ac.in.

D. Mukherjee is with Department of Electrical Engineering, Indian Institute of Technology Bombay, Powai- 400076, Mumbai, India. e-mail: dm@ee.iitb.ac.in. 
Pioneering work in impact time guidance can be attributed to [7] wherein a biased proportional-navigation guidance (PNG) strategy was used by combining PNG with a feedback of impact time error, derived using a linearized engagement geometry. Impact time control necessitates that time-to-go, a quantity associated with flight conditions in future, be known a priori. However, obtaining a closed form expression for the same is not a trivial task [8]. A time-to-go estimate taking interceptor's heading angle into account was presented in [9], and was widely used in conjunction with small angle assumption. The work in [10] provided an extension to some special classes of impact time guidance strategies using a time-to-go prediction formula and a desired error dynamics.

With rising interest in time-constrained guidance over the years, many advanced guidance strategies have been proposed using Lyapunov methods and sliding mode control (SMC) [11-14]. The guidance strategy in [11] required the interceptor look angle to decrease monotonically during the engagement to satisfy impact time requirements. Note that such a variation in look angle may not lead to a wide range of achievable impact time values. The work in [12] achieved a larger impact time interval by considering large heading angle errors and even interceptor's negative closing speed. Authors in [13] reported simplifications of the sliding surface to design an impact time guidance strategy and handled the singularities in the guidance command by suitable modification. By considering an improved time-to-go estimate, without small angle assumptions, it was demonstrated in [14] that significantly wider range of impact time values can be achieved for arbitrary initial heading of the interceptor.

The aforementioned results were primarily obtained against stationary targets and they can be extended to intercept constant speed targets using the concept of predicted interception point (PIP) [9,12,14]. Approximations, such as PIP, are susceptible to errors in impact time, which may lead to a miss. Moreover, since the time-to-go expressions used to derive the guidance commands in the aforementioned studies were mere estimates, it is likely that the initial value of the same might be erroneous. An impact time guidance strategy, for both 2D and 3D engagements against a moving target, was proposed in [15] wherein conventional time-to-go estimate was used to derive the guidance command. Under large heading errors, conventional time-to-go estimates may become less accurate, eventually leading to error in impact time. In [16], the impact time guidance strategy was designed against non-stationary targets without using explicit time-to-go, by constructing a sliding manifold as a weighted sum of the relative range and the desired time-to-go. The weighting functions in [16] were polynomial-based, which may impose computational burden on the guidance system if the order of polynomial is increased. The time-constrained strategy proposed in [17] utilized deviated pursuit guidance [18], originally developed to intercept constant speed targets, making PIP approximations unnecessary. The deviated pursuit guidance also offers an exact expression of time-to-go from the beginning of the engagement, thereby nullifying the chances of errors that creep in due to its estimation.

The above guidance strategies were designed considering engagement kinematics only, and neglect the interceptor dynamics and actuators involved. However, in practice, the desired lateral acceleration cannot be achieved instantaneously by the interceptor. While designing the outer guidance loop and inner control loop separately simplifies the overall design process, it has been shown in [3-6] that an integrated design of guidance and control (IGC) outperforms separate two-loop designs. IGC design using sliding mode control have been reported in [19,20] wherein the studies demonstrated improvements in the performance of the homing interceptor in terms of smaller response time, 
reduced miss distance and better dynamic behavior. A comprehensive Monte Carlo simulation was presented in [21] to demonstrate the effectiveness of IGC design over separate two-loop ones. In [22], the integrated design was studied from the perspective of optimal control formulations, wherein the autopilot design and the interceptor's guidance law were formulated in a unified state space framework. Integrated guidance and control was also found to be effective in satisfying terminal angle constraints $[23,24]$. It should be noted that although effective, the above mentioned studies used only a single control surface, either a canard or a tail, to generate the necessary lateral acceleration. However, recent studies, $[25,26]$, have shown that by using both canard and tail control configurations, not only superior guidance performance can be realized, but the additional degree of freedom may also be used to achieve additional objectives.

Motivated by the efficacy of integrated guidance and control in satisfying terminal angle constraints, it stands to reason that other important terminal constraints, such as impact time, can also be met. To the best of the authors' knowledge, integrated guidance and control to meet impact time requirement has not been reported in the literature so far. Towards that end, the contributions of this work are five fold.

- The complete design, against a non-maneuvering target, is carried out in nonlinear settings. Consequently, possible errors due to linearization of the kinematics and dynamics, using small angle assumptions, are avoided. The proposed strategies remain applicable even for the engagements with large initial heading angle errors.

- The time-to-go expression used in deriving the guidance commands are based on an improved estimate in the case of stationary targets, and an exact one in the case of constant speed targets. This, naturally, allows the proposed strategies to remain effective for wide interval of impact time.

- Design of the proposed strategies utilizes sliding mode control, which offers ease of design and insensitivity to matched anomalies.

- A weighted allocation technique has been proposed to apportion the required effort via canard and tail deflections. By adjusting the weights, suitable deflections, and in turn, required lateral acceleration can be generated.

- Actuator dynamics have also been considered while designing the proposed strategies, and time-constrained interception in the event of actuator failure is also briefly discussed.

The remainder of this paper is organized as follows. Section 2 presents the nonlinear engagement kinematics and the interceptor dynamics, along with the main problem addressed in this work, followed by the derivation of the integrated guidance and control strategies in Section 3. Section 4 presents simulations for various cases to support the theory, and Section 5 concludes the paper with some directions for future investigations. 


\section{Problem formulation}

Consider the planar engagement geometry between an endo-atmospheric interceptor and a target as shown in Fig. 1, whose kinematics is governed by the set of nonlinear equations given by

$$
\begin{gathered}
\dot{r}=V_{\mathrm{T}} \cos \left(\gamma_{\mathrm{T}}-\theta\right)-V_{\mathrm{M}} \cos \sigma=V_{r}, \\
r \dot{\theta}=V_{\mathrm{T}} \sin \left(\gamma_{\mathrm{T}}-\theta\right)-V_{\mathrm{M}} \sin \sigma=V_{\theta}, \\
\dot{\gamma}_{\mathrm{M}}=a_{\mathrm{M}} / V_{\mathrm{M}}, \dot{\gamma}_{\mathrm{T}}=a_{\mathrm{T}} / V_{\mathrm{T}}, \sigma=\gamma_{\mathrm{M}}-\theta,
\end{gathered}
$$

where $V_{\mathrm{M}}, V_{\mathrm{T}}$ denote the speed of the interceptor and the target, respectively, while $\gamma_{\mathrm{M}}$ and $\gamma_{\mathrm{T}}$ are their respective flight path angles. The relative distance and the line-of-sight (LOS) between the adversaries are represented by $r$ and $\theta$, respectively, while the interceptor's look angle is denoted by $\sigma$. The components of relative velocities along and across the LOS are $V_{r}$ and $V_{\theta}$, respectively, while the lateral accelerations of the interceptor and the target are $a_{\mathrm{M}}$ and $a_{\mathrm{T}}$, respectively. It is assumed that the interceptor and the target possess only lateral acceleration capabilities, with their speeds being constant throughout the engagement.

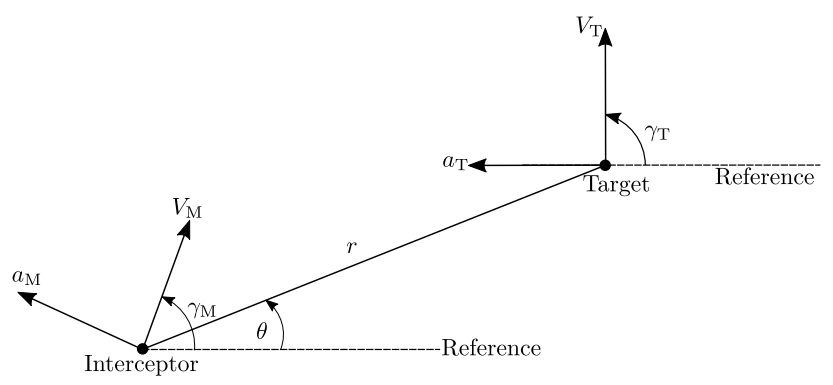

Figure 1: Planar engagement geometry.

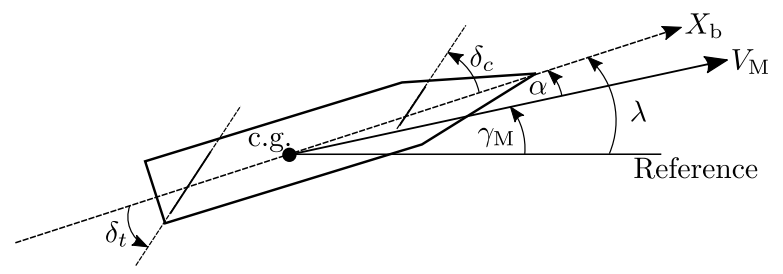

Figure 2: Planar interceptor dynamics.

For an interceptor steered by the combined effects of various aerodynamic surfaces, as shown in Fig. 2, the planar dynamics, in the pitch plane [25], is governed by

$$
\begin{aligned}
\dot{\alpha} & =q-L\left(\alpha, \delta_{c}, \delta_{t}\right) /\left(m V_{\mathrm{M}}\right)=q-a_{\mathrm{M}} / V_{\mathrm{M}}, \\
\dot{q} & =M\left(\alpha, q, \delta_{c}, \delta_{t}\right) / I, \dot{\lambda}=q, \\
\dot{\delta}_{c} & =\left(\delta_{c}^{c}-\delta_{c}\right) / \tau_{c}, \dot{\delta}_{t}=\left(\delta_{t}^{c}-\delta_{t}\right) / \tau_{t},
\end{aligned}
$$

where $\alpha, \lambda$, and $q$ denote the angle of attack, pitch angle, and the pitch rate, respectively. The lift and the aerodynamic 
pitch moment acting on the interceptor are represented by $L(\cdot)$ and $M(\cdot)$, respectively, while $m$ and $I$, are respective representations of interceptor's mass and moment of inertia. It is assumed that the interceptor's tail and canard, which are the actuators, are described by first-order dynamics with time constants $\tau_{c}$ and $\tau_{t}$, respectively. The deflection of the canard is represented by $\delta_{c}$ while that of the tail is denoted by $\delta_{t}$. The commanded deflections, $\delta_{c}^{c}$ and $\delta_{t}^{c}$, act as control inputs for the aerodynamic surfaces, the canard and the tail, respectively.

The lift forces and the aerodynamic pitch moments generated by the interceptor's body and the control surfaces can be described by

$$
\begin{aligned}
& L / m=L_{\alpha}^{\beta} g_{1}(\alpha)+L_{\delta_{c}} g_{2}\left(\alpha+\delta_{c}\right)+L_{\delta_{t}} g_{3}\left(\alpha+\delta_{t}\right), \\
& M / I=M_{q} q+M_{\alpha}^{\beta} g_{4}(\alpha)+M_{\delta_{c}} g_{5}\left(\alpha+\delta_{c}\right)+M_{\delta_{t}} g_{6}\left(\alpha+\delta_{t}\right),
\end{aligned}
$$

where $L_{\alpha}^{\beta}, L_{\delta_{c}}, L_{\delta_{t}}$ are the lift coefficients, and $M_{q}, M_{\alpha}^{\beta}, M_{\delta_{c}}, M_{\delta_{t}}$ are the moment coefficients. The functions $g_{i}(\cdot) \forall i=$ $1, \ldots, 6$ are assumed to be smooth, bounded, and nonlinear functions, describing the aerodynamic characteristics of the interceptor. The interceptor's lateral acceleration is related to aerodynamics forces (lift) as $a_{\mathrm{M}}=L / m$, and is given by (3).

Here we are concerned with terminal constraints on the interceptor's trajectory, especially a specified impact time. Impact time, $T_{f}$, is defined as the total time at which the interceptor intercepts the target, which is the sum of the time elapsed post its launch, $t_{\mathrm{el}}$, and the time remaining from that instant until interception, also known as time-to-go, $t_{\mathrm{go}},[12]$.

The main problem addressed in this paper is to design integrated guidance commands for the interceptor, which enables a time-constrained interception of a non-maneuvering target. The guidance commands, or the canard and the tail deflections, should provide necessary lateral acceleration to steer the endo-atmospheric interceptor on a course requisite for a time-constrained interception. It is also important that the proposed strategies remain effective for engagements with large heading errors, allowing the interceptor to achieve a wide range of impact time.

\section{Integrated Guidance and Control Design}

In this section, we derive the necessary commands to satisfy the guidance objectives. First, we obtain the relation among the dynamics of interceptor's lateral acceleration, aerodynamic forces, surface deflections, and other parameters by differentiating (3), as

$$
\begin{aligned}
\dot{a}_{\mathrm{M}} & =L_{\alpha}^{\beta} g_{1}^{\prime}(\alpha) \dot{\alpha}+L_{\delta_{c}}\left(\dot{\alpha}+\dot{\delta}_{c}\right) g_{2}^{\prime}\left(\alpha+\delta_{c}\right)+L_{\delta_{t}}\left(\dot{\alpha}+\dot{\delta}_{t}\right) g_{3}^{\prime}\left(\alpha+\delta_{t}\right) \\
& =\left[L_{\alpha}^{\beta} g_{1}^{\prime}(\alpha)+L_{\delta_{c}} g_{2}^{\prime}\left(\alpha+\delta_{c}\right)+L_{\delta_{t}} g_{3}^{\prime}\left(\alpha+\delta_{t}\right)\right] \dot{\alpha}+\frac{L_{\delta_{c}}\left(\delta_{c}^{c}-\delta_{c}\right) g_{2}^{\prime}\left(\alpha+\delta_{c}\right)}{\tau_{c}}+\frac{L_{\delta_{t}}\left(\delta_{t}^{c}-\delta_{t}\right) g_{3}^{\prime}\left(\alpha+\delta_{t}\right)}{\tau_{t}} \\
& =\Gamma q-\Gamma \frac{a_{\mathrm{M}}}{V_{\mathrm{M}}}-\left[\frac{L_{\delta_{c}}}{\tau_{c}} g_{2}^{\prime}\left(\alpha+\delta_{c}\right) \delta_{c}+\frac{L_{\delta_{t}}}{\tau_{t}} g_{3}^{\prime}\left(\alpha+\delta_{t}\right) \delta_{t}\right]+\frac{L_{\delta_{c}}}{\tau_{c}} g_{2}^{\prime}\left(\alpha+\delta_{c}\right) \delta_{c}^{c}+\frac{L_{\delta_{t}^{c}}}{\tau_{t}} g_{3}^{\prime}\left(\alpha+\delta_{t}\right) \delta_{t}^{c},
\end{aligned}
$$


where $\Gamma=\left[L_{\alpha}^{\beta} g_{1}^{\prime}(\alpha)+L_{\delta_{c}} g_{2}^{\prime}\left(\alpha+\delta_{c}\right)+L_{\delta_{t}} g_{3}^{\prime}\left(\alpha+\delta_{t}\right)\right]$. It is worth noting that both the aerodynamic surface deflection commands, $\delta_{c}^{c}$ and $\delta_{t}^{c}$, have relative degree one with respect to the dynamics of interceptor's lateral acceleration.

For subsequent design of IGC strategies enabling a time-constrained interception against a non-maneuvering target, the error in time of interception is defined as

$$
e_{t}=t_{\mathrm{go}}-t_{\mathrm{go}}^{\mathrm{d}}=t_{\mathrm{go}}-T_{f}+t_{\mathrm{el}}
$$

where $t_{\text {go }}^{\mathrm{d}}$ is the desired time-to-go and $\dot{t}_{\mathrm{go}}^{\mathrm{d}}=-\dot{t}_{\mathrm{el}}=-1$.

The integrated guidance and control design against a stationary target is delineated first, followed by the same against a constant speed target.

\subsection{Stationary Targets}

For a stationary target, $V_{\mathrm{T}}$ is zero, reducing the relative velocities, (1a)-(1b), in the engagement kinematics to $\dot{r}=$ $-V_{\mathrm{M}} \cos \sigma$ and $r \dot{\theta}=-V_{\mathrm{M}} \sin \sigma$. For a stationary target, the time-to-go estimate accounting for interceptor's heading angle, [9], without any small angle assumption, is given by

$$
t_{\mathrm{go}}=\frac{r}{V_{\mathrm{M}}}\left(1+\frac{\sin ^{2} \sigma}{K}\right) ; K=4 N-2
$$

where $N$ is the navigation constant in the notions of PNG. It is worth noting from (7) that an interception is guaranteed if and only if the time-to-go estimate, (7), is zero. We first present some intermediate results that aid in the design process. On differentiating (6) with respect to time, one may obtain

$$
\dot{e}_{t}=\dot{t}_{\mathrm{go}}+1
$$

which can be further simplified by differentiating (7) once, as

$$
\begin{aligned}
\dot{e}_{t} & =1-\frac{\dot{r}}{V_{\mathrm{M}}}\left(1+\frac{\sin ^{2} \sigma}{K}\right)+\frac{r}{V_{\mathrm{M}}}\left(\frac{\sin 2 \sigma}{K}\right) \dot{\sigma}=1-\cos \sigma\left(1+\frac{\sin ^{2} \sigma}{K}\right)-\left(\frac{r \dot{\theta} \sin 2 \sigma}{K V_{\mathrm{M}}}\right)+\left(\frac{r \sin 2 \sigma}{K V_{\mathrm{M}}^{2}}\right) a_{\mathrm{M}} \\
& =-\cos \sigma\left(1-\frac{\sin ^{2} \sigma}{K}\right)+\left(\frac{r \sin 2 \sigma}{K V_{\mathrm{M}}^{2}}\right) a_{\mathrm{M}},
\end{aligned}
$$

from which it is immediate that the time-to-go error of interceptor-target engagement has a relative degree one with respect to the interceptor's lateral acceleration. It follows from (5) that $\delta_{c}^{c}$ and $\delta_{t}^{c}$ have relative degree of one with respect to $a_{\mathrm{M}}$. Hence, differentiating (9) once more gives a relation between the time-to-go error and the aerodynamic surface deflections as

$$
\ddot{e}_{t}=\left[1+\frac{\left(2-3 \sin ^{2} \sigma\right)}{K}\right] \frac{V_{\mathrm{M}} \sin ^{2} \sigma}{r}+\frac{2 r \cos 2 \sigma}{K V_{\mathrm{M}}^{3}} a_{\mathrm{M}}^{2}+\left[1+\frac{\left(2-5 \sin ^{2} \sigma\right)}{K}\right] \frac{\sin \sigma}{V_{\mathrm{M}}} a_{\mathrm{M}}+\frac{r \sin 2 \sigma}{K V_{\mathrm{M}}^{2}} \dot{a}_{\mathrm{M}},
$$


which can be further simplified using (5) as

$$
\begin{aligned}
\ddot{e}_{t}= & {\left[1+\frac{\left(2-3 \sin ^{2} \sigma\right)}{K}\right] \frac{V_{\mathrm{M}} \sin ^{2} \sigma}{r}+\frac{2 r \cos 2 \sigma}{K V_{\mathrm{M}}^{3}} a_{\mathrm{M}}^{2}+\left[1+\frac{\left(2-5 \sin ^{2} \sigma\right)}{K}\right] \frac{\sin \sigma}{V_{\mathrm{M}}} a_{\mathrm{M}}+\frac{r \sin 2 \sigma}{K V_{\mathrm{M}}^{2}}[\Gamma q} \\
& \left.-\Gamma \frac{a_{\mathrm{M}}}{V_{\mathrm{M}}}-\frac{L_{\delta_{c}}}{\tau_{c}} g_{2}^{\prime}\left(\alpha+\delta_{c}\right) \delta_{c}-\frac{L_{\delta_{t}}}{\tau_{t}} g_{3}^{\prime}\left(\alpha+\delta_{t}\right) \delta_{t}+\frac{L_{\delta_{c}}}{\tau_{c}} g_{2}^{\prime}\left(\alpha+\delta_{c}\right) \delta_{c}^{c}+\frac{L_{\delta_{t}^{c}}}{\tau_{t}} g_{3}^{\prime}\left(\alpha+\delta_{t}\right) \delta_{t}^{c}\right] .
\end{aligned}
$$

Thus, the time-to-go error of interceptor-target engagement has a relative degree of two with respect to the control surface deflections.

We shall use the notions of SMC to design the IGC strategies, with the central idea being the confinement of the system trajectories on a reduced order subspace/manifold.. This offers alluring features such as suppression of disturbances, desired dynamical behavior and tractability of design, for various applications [12-14,27]. The significance of the relative degrees of $e_{t}$ and $\dot{e}_{t}$ are apparent upon constructing a sliding manifold of the form

$$
s=\dot{e}_{t}+c e_{t}
$$

where $c>0$ is a parameter governing the rate of convergence of $e_{t}$ during sliding mode. Differentiating (11) with respect to time, and using (9) and (10), yield

$$
\dot{s}=\ddot{e}_{t}+c \dot{e}_{t}=\ddot{t}_{\mathrm{go}}+c \dot{t}_{\mathrm{go}}+c=\mathcal{F}+\mathcal{B}_{c} \delta_{c}^{c}+\mathcal{B}_{t} \delta_{t}^{c}
$$

where

$$
\begin{aligned}
\mathcal{F}= & {\left[1+\frac{\left(2-3 \sin ^{2} \sigma\right)}{K}\right] \frac{V_{\mathrm{M}} \sin ^{2} \sigma}{r}+\frac{2 r \cos 2 \sigma}{K V_{\mathrm{M}}^{3}} a_{\mathrm{M}}^{2}+\left[1+\frac{\left(2-5 \sin ^{2} \sigma\right)}{K}\right] \frac{\sin \sigma}{V_{\mathrm{M}}} a_{\mathrm{M}}+\frac{r \sin 2 \sigma}{K V_{\mathrm{M}}^{2}}[\Gamma q} \\
& \left.-\Gamma \frac{a_{\mathrm{M}}}{V_{\mathrm{M}}}-\frac{L_{\delta_{c}}}{\tau_{c}} g_{2}^{\prime}\left(\alpha+\delta_{c}\right) \delta_{c}-\frac{L_{\delta_{t}}}{\tau_{t}} g_{3}^{\prime}\left(\alpha+\delta_{t}\right) \delta_{t}\right]+c\left[1-\cos \sigma\left(1-\frac{\sin ^{2} \sigma}{K}\right)+\left(\frac{r \sin 2 \sigma}{K V_{\mathrm{M}}^{2}}\right) a_{\mathrm{M}}\right], \\
\mathcal{B}_{c}= & \frac{L_{\delta_{c}} g_{2}^{\prime}\left(\alpha+\delta_{c}\right) r \sin 2 \sigma}{K V_{\mathrm{M}}^{2} \tau_{c}}, \mathcal{B}_{t}=\frac{L_{\delta_{t}} g_{3}^{\prime}\left(\alpha+\delta_{t}\right) r \sin 2 \sigma}{K V_{\mathrm{M}}^{2} \tau_{t}} .
\end{aligned}
$$

It is apparent from (10) and (12) that the sliding manifold, (11), is influenced by both $\delta_{c}^{c}$ and $\delta_{t}^{c}$. The time-to-go tracks its desired value after the occurrence of sliding mode, as described in the next theorem.

Theorem 1. For a planar engagement between an endo-atmospheric interceptor and a stationary target, whose engagement kinematics is governed by (1), with the time-to-go error, (6), and the sliding manifold, (11), a time-constrained interception is guaranteed to occur at a desired time, $T_{f}$, if the control surface deflections are chosen to satisfy the relation

$$
\mathcal{B}_{c} \delta_{c}^{c}+\mathcal{B}_{t} \delta_{t}^{c}=-\mathcal{F}-\mu \operatorname{sign}(s), \mu>0
$$

Proof. By considering a Lyapunov function candidate, $\mathcal{V}=s^{2} / 2$, and differentiating it with respect to time, one obtains

$$
\dot{\mathcal{V}}=s \dot{s}=s\left(\ddot{e}_{t}+c \dot{e}_{t}\right)=s\left(\mathcal{F}+\mathcal{B}_{c} \delta_{c}^{c}+\mathcal{B}_{t} \delta_{t}^{c}\right)
$$


Choosing $\mathcal{B}_{c} \delta_{c}^{c}+\mathcal{B}_{t} \delta_{t}^{c}=-\mathcal{F}-\mu \operatorname{sign}(s)$ renders the dynamics of the sliding manifold, (12), as $\dot{s}=-\mu \operatorname{sign}(s)$. Hence, (16) simplifies to $\dot{\mathcal{V}}=-\mu|s|$, which is negative for any $\mu>0$, resulting in the stability of the sliding mode dynamics.

After sliding mode is enforced, (11) becomes zero. As a result, the error, $e_{t}$, exhibits an exponential decay whose rate is governed by the parameter $c$. In other words, $e_{t}(t)=e_{t}\left(t_{s}\right) \mathrm{e}^{-c\left(t-t_{s}\right)}$, for all $t>t_{s}$, where $t_{s}<T_{f}$ is the time when sliding mode occurs. Therefore, the interceptor's time-to-go tracks the desired value asymptotically, resulting in a time-constrained interception at a desired time, $T_{f}$. This concludes the proof.

From the expression of $\mathcal{V}$, one can write $s=\sqrt{2 \mathcal{V}}$, and therefore, $\dot{\mathcal{V}}=-\mu \mid \sqrt{2 \mathcal{V}}$. The reaching time, $t_{s}$, can be determined by evaluating

$$
\int_{\mathcal{V}(0)}^{0} \frac{\mathrm{d} \mathcal{V}}{|\sqrt{2 \mathcal{V}}|}=-\int_{0}^{t_{s}} \mu \mathrm{d} t
$$

which results in $t_{s}=\frac{\sqrt{2 \mathcal{V}(0)}}{\mu}=\frac{s(0)}{\mu}$. This shows that sliding mode is enforced on the manifold, (11), in a finite time, $t_{s}$.

It can be inferred from (12) and Theorem 1 that there exist various ways to enforce sliding mode, that is, there are infinitely many choices of $\delta_{c}^{c}$ and $\delta_{t}^{c}$ which ensure a time-constrained interception at a desired time. One possibility is to use either of the aerodynamic surfaces while neglecting the other. In practice, the capabilities of both the aerodynamic surfaces may not be similar. Thus, it might be more meaningful to suitably allocate the available effort in both the aerodynamic surfaces depending on their capabilities. In what follows next, we present a general design procedure of weighted allocation that provides flexibility in apportioning the available effort by considering a cost function of the form

$$
\mathcal{J}=\sqrt{\left(\frac{\delta_{c}^{c}}{w_{c}}\right)^{2}+\left(\frac{\delta_{t}^{c}}{w_{t}}\right)^{2}},
$$

where $w_{c}>0, w_{t}>0$ are weighting parameters. The cost function, (17), can be thought of as instantaneous minimization of the overall aerodynamic surface deflection. Note that we do not aim for overall minimization of the control effort throughout the engagement. The expressions for the proposed IGC commands are presented in the next theorem.

Theorem 2. For a planar engagement between an endo-atmospheric interceptor and a stationary target, whose engagement kinematics is governed by (1), with the dynamics of the interceptor described by (2) and the sliding manifold chosen as (11), the proposed integrated guidance and control commands

$$
\begin{aligned}
\delta_{c}^{c}= & -\frac{L_{\delta_{c}} g_{2}^{\prime}\left(\alpha+\delta_{c}\right) \tau_{c} \tau_{t}^{2}}{L_{\delta_{c}}^{2} \tau_{t}^{2} g_{2}^{\prime 2}\left(\alpha+\delta_{c}\right)+L_{\delta_{t}}^{2} \tau_{c}^{2} g_{3}^{\prime 2}\left(\alpha+\delta_{t}\right) w^{2}}\left[\frac{2 a_{\mathrm{M}}^{2}}{V_{\mathrm{M}} \tan 2 \sigma}+\frac{V_{\mathrm{M}}\left(K+2-5 \sin ^{2} \sigma\right)}{2 r \cos \sigma} a_{\mathrm{M}}+\frac{V_{\mathrm{M}}^{3} \tan \sigma}{2 r^{2}}(K+2\right. \\
& \left.-3 \sin ^{2} \sigma\right)+\Gamma q-\Gamma \frac{a_{\mathrm{M}}}{V_{\mathrm{M}}}-\frac{L_{\delta_{c}}}{\tau_{c}} g_{2}^{\prime}\left(\alpha+\delta_{c}\right) \delta_{c}-\frac{L_{\delta_{t}}}{\tau_{t}} g_{3}^{\prime}\left(\alpha+\delta_{t}\right) \delta_{t}-\frac{c K V_{\mathrm{M}}^{2} \tan \sigma / 2}{2 r \cos \sigma}+\frac{c V_{\mathrm{M}}^{2} \sin \sigma}{2 r} \\
& \left.+c a_{\mathrm{M}}+\frac{K V_{\mathrm{M}}^{2}}{r \sin 2 \sigma} \mu \operatorname{sign}(s)\right]
\end{aligned}
$$




$$
\begin{aligned}
\delta_{t}^{c}= & -\frac{L_{\delta_{t}} g_{3}^{\prime}\left(\alpha+\delta_{t}\right) \tau_{t} \tau_{c}^{2} w^{2}}{L_{\delta_{c}}^{2} \tau_{t}^{2} g_{2}^{\prime 2}\left(\alpha+\delta_{c}\right)+L_{\delta_{t}}^{2} \tau_{c}^{2} g_{3}^{\prime 2}\left(\alpha+\delta_{t}\right) w^{2}}\left[\frac{2 a_{\mathrm{M}}^{2}}{V_{\mathrm{M}} \tan 2 \sigma}+\frac{V_{\mathrm{M}}\left(K+2-5 \sin ^{2} \sigma\right)}{2 r \cos \sigma} a_{\mathrm{M}}+\frac{V_{\mathrm{M}}^{3} \tan \sigma}{2 r^{2}}(K+2\right. \\
& \left.-3 \sin ^{2} \sigma\right)+\Gamma q-\Gamma \frac{a_{\mathrm{M}}}{V_{\mathrm{M}}}-\frac{L_{\delta_{c}}}{\tau_{c}} g_{2}^{\prime}\left(\alpha+\delta_{c}\right) \delta_{c}-\frac{L_{\delta_{t}}}{\tau_{t}} g_{3}^{\prime}\left(\alpha+\delta_{t}\right) \delta_{t}-\frac{c K V_{\mathrm{M}}^{2} \tan \sigma / 2}{2 r \cos \sigma}+\frac{c V_{\mathrm{M}}^{2} \sin \sigma}{2 r} \\
& \left.+c a_{\mathrm{M}}+\frac{K V_{\mathrm{M}}^{2}}{r \sin 2 \sigma} \mu \operatorname{sign}(s)\right],
\end{aligned}
$$

minimize the cost function, (17), subject to the constraint (15), where $\mathcal{F}, \mathcal{B}_{c}$ and $\mathcal{B}_{t}$ are given by (13)-(14). Further, this leads to the interceptor attaining a course requisite for time-constrained interception within a finite-time, $t_{s}$, and guarantees target interception at a desired time, $T_{f}$.

Proof. It follows from Theorem 1 that sliding mode is enforced within $t_{s}$ if (15) is satisfied. By treating $\mathcal{B}_{c} \delta_{c}^{c}+\mathcal{B}_{t} \delta_{t}^{c}=$ $-\mathcal{F}-\mu \operatorname{sign}(s)=\Upsilon$, in (15), as a constraint in the minimization of the cost, (17), one can write $\delta_{t}^{c}=\frac{\Upsilon-\mathcal{B}_{c} \delta_{c}^{c}}{\mathcal{B}_{t}}$ which, upon substituting in (17), yields

$$
\mathcal{J}=\left[\left(\frac{\delta_{c}^{c}}{w_{c}}\right)^{2}+\left(\frac{\Upsilon-\mathcal{B}_{c} \delta_{c}^{c}}{\mathcal{B}_{t} w_{t}}\right)^{2}\right]^{1 / 2}
$$

The solution to $\frac{\partial \mathcal{J}}{\partial \delta_{c}^{c}}=0$, with (15) as a constraint, results in

$$
\delta_{c}^{c}=\frac{\mathcal{B}_{c} \Upsilon}{\mathcal{B}_{c}^{2}+\mathcal{B}_{t}^{2} w^{2}}, \delta_{t}^{c}=\frac{\mathcal{B}_{t} \Upsilon w^{2}}{\mathcal{B}_{c}^{2}+\mathcal{B}_{t}^{2} w^{2}}
$$

where $w=w_{t} / w_{c}$. It can be readily verified that the values corresponding to the aerodynamic surface deflections, in (21), are minimum, by evaluating $\frac{\partial^{2} \mathcal{J}}{\partial \delta_{c}^{c^{2}}}$ at these values. Substituting the values of $\Upsilon, \mathcal{B}_{c}$ and $\mathcal{B}_{t}$, obtained in this part, in (21), the expressions for the canard and the tail surface deflections, given in (18)-(19) can be obtained. This concludes the proof.

\subsection{Constant Speed Targets}

The engagement geometry for this case remains the same as in (1). The time-to-go estimate, (7), albeit good against stationary targets, may be prone to error when PIP is used to intercept a constant speed target. In other words, if there are errors in the estimation of time-to-go, PIP approximation might lead to a miss. This motivates us to resort to a technique that was originally developed to intercept moving targets- use of deviated pursuit. The expression of time-to-go for an interceptor guided by deviated pursuit, with a fixed deviation angle $\sigma$, is given by

$$
t_{\mathrm{go}}=\frac{r}{\eta}\left[V_{r}+2 V_{\mathrm{M}} \cos \sigma-V_{\theta} \tan \sigma\right]=\frac{r \sec \sigma}{\eta}\left[V_{\mathrm{M}}+V_{\mathrm{T}} \cos \left(\gamma_{\mathrm{T}}-\theta+\sigma\right)\right],
$$

where $\eta=V_{\mathrm{M}}^{2}-V_{\mathrm{T}}^{2}$. Note that the expression in $(22)$ is exact $[17,18]$ and does not involve any approximation or small angle assumption, thus eliminating errors arising due to time-to-go estimation and small angle approximations. Consequently, the proposed design remains effective in engagements that may require the interceptor's heading error to be large. From (22), it is immediate that $t_{\mathrm{go}}=0 \Longleftrightarrow r=0$, as shown in [17]. This observation is similar to that in the previous case of stationary target interception. To derive the IGC commands for interception of a constant speed 
target, we proceed by computing the derivatives of the time-to-go error, (6), using the expression of time-to-go, (22), as

$$
\begin{aligned}
\dot{e}_{t} & =1+\dot{t}_{\mathrm{go}}=1+\frac{1}{\eta^{2}}\left[V_{r}\left(V_{r}+2 V_{\mathrm{M}} \cos \sigma-V_{\theta} \tan \sigma\right)+r\left(\dot{V}_{r}-2 V_{\mathrm{M}} \sin \sigma \dot{\sigma}-\dot{V}_{\theta} \tan \sigma-V_{\theta} \sec ^{2} \sigma \dot{\sigma}\right)\right] \\
& =\frac{V_{\theta}^{2} \sec ^{2} \sigma}{\eta}-\frac{r V_{\theta} \sec ^{2} \sigma}{V_{\mathrm{M}} \eta} a_{\mathrm{M}},
\end{aligned}
$$

where $\dot{V}_{r}=\frac{V_{\theta}^{2}}{r}+a_{\mathrm{M}} \sin \sigma$ and $\dot{V}_{\theta}=-\frac{V_{r} V_{\theta}}{r}-a_{\mathrm{M}} \cos \sigma$ were used. Similarly, $\ddot{e}_{t}=\ddot{t}_{\mathrm{go}}$ can be obtained by differentiating (23) with respect to time as

$$
\ddot{e}_{t}=\frac{2 V_{\theta} \dot{V}_{\theta} \sec ^{2} \sigma}{\eta}-\frac{2 V_{\theta}^{3} \sec ^{2} \sigma \tan \sigma}{r \eta}-\frac{2 r V_{\theta} \sec ^{2} \sigma \tan \sigma}{V_{\mathrm{M}}^{2} \eta} a_{\mathrm{M}}^{2}+\frac{\sec ^{2} \sigma}{V_{\mathrm{M}} \eta}\left[4 V_{\theta}^{2} \tan \sigma-V_{r} V_{\theta}-r \dot{V}_{\theta}\right] a_{\mathrm{M}}-\frac{r V_{\theta} \sec ^{2} \sigma}{V_{\mathrm{M}} \eta} \dot{a}_{\mathrm{M}}
$$

which can be further simplified by substituting (5) as

$$
\begin{aligned}
\ddot{e}_{t}= & \frac{2 V_{\theta}}{\eta \cos ^{2} \sigma}\left[\dot{V}_{\theta}-\frac{V_{\theta}^{2} \tan \sigma}{r}\right]-\frac{2 r V_{\theta} \sec ^{2} \sigma \tan \sigma}{V_{\mathrm{M}}^{2} \eta} a_{\mathrm{M}}^{2}+\frac{\sec ^{2} \sigma}{V_{\mathrm{M}} \eta}\left[4 V_{\theta}^{2} \tan \sigma-V_{r} V_{\theta}-r \dot{V}_{\theta}\right] a_{\mathrm{M}}-\frac{r V_{\theta} \sec ^{2} \sigma}{V_{\mathrm{M}} \eta}[\Gamma q \\
& \left.-\Gamma \frac{a_{\mathrm{M}}}{V_{\mathrm{M}}}-\frac{L_{\delta_{c}}}{\tau_{c}} g_{2}^{\prime}\left(\alpha+\delta_{c}\right) \delta_{c}-\frac{L_{\delta_{t}}}{\tau_{t}} g_{3}^{\prime}\left(\alpha+\delta_{t}\right) \delta_{t}+\frac{L_{\delta_{c}}}{\tau_{c}} g_{2}^{\prime}\left(\alpha+\delta_{c}\right) \delta_{c}^{c}+\frac{L_{\delta_{t}^{c}}}{\tau_{t}} g_{3}^{\prime}\left(\alpha+\delta_{t}\right) \delta_{t}^{c}\right] .
\end{aligned}
$$

It can be inferred from (23) and (24) that the relative degrees of the time-to-go error, (6), with respect to the lateral acceleration and the aerodynamic surface deflections are one and two, respectively. These observations are similar to those in the case of stationary targets.

As a result, the same sliding manifold, (11), can be used to derive the integrated guidance and control commands, and indicate that the essence of Theorem 1 remains valid in this case. However, $\mathcal{F}, \mathcal{B}_{c}$ and $\mathcal{B}_{t}$, for this case are different from those obtained in (13)-(14), as they depend on (22)-(24), and can be determined by substituting (23) and (24) in (12). The following theorem summarizes this and is stated without proof since its proof is similar to that of Theorem 2.

Theorem 3. For a planar engagement between an endo-atmospheric interceptor and a constant speed target, whose engagement kinematics is governed by (1), with the time-to-go error, (6), and the sliding manifold, (11), a time-constrained interception at a desired time, $T_{f}$, is guaranteed to occur if the relation in (15) holds for chosen surface deflections, where

$$
\begin{aligned}
\mathcal{F}= & \frac{2 V_{\theta}}{\eta \cos ^{2} \sigma}\left[\dot{V}_{\theta}-\frac{V_{\theta}^{2} \tan \sigma}{r}\right]-\frac{2 r V_{\theta} \sec ^{2} \sigma \tan \sigma}{V_{\mathrm{M}}^{2} \eta} a_{\mathrm{M}}^{2}+\frac{\sec ^{2} \sigma}{V_{\mathrm{M}} \eta}\left[4 V_{\theta}^{2} \tan \sigma-V_{r} V_{\theta}-r \dot{V}_{\theta}\right] a_{\mathrm{M}}-\frac{r V_{\theta} \sec ^{2} \sigma}{V_{\mathrm{M}} \eta}[\Gamma q \\
& -\Gamma \frac{a_{\mathrm{M}}}{V_{\mathrm{M}}}-\frac{L_{\delta_{c}}}{\tau_{c}} g_{2}^{\prime}\left(\alpha+\delta_{c}\right) \delta_{c}-\frac{L_{\delta_{t}}}{\tau_{t}} g_{3}^{\prime}\left(\alpha+\delta_{t}\right) \delta_{t}-\frac{c V_{\theta} \sec ^{2} \sigma}{\eta}\left(V_{\theta}-\frac{r}{V_{\mathrm{M}}} a_{\mathrm{M}}\right), \\
\mathcal{B}_{c}= & \frac{L_{\delta_{c}} g_{2}^{\prime}\left(\alpha+\delta_{c}\right) r V_{\theta}}{\tau_{c} V_{\mathrm{M}} \eta \cos ^{2} \sigma}, \mathcal{B}_{t}=\frac{L_{\delta_{t}} g_{3}^{\prime}\left(\alpha+\delta_{t}\right) r V_{\theta}}{\tau_{t} V_{\mathrm{M}} \eta \cos ^{2} \sigma} .
\end{aligned}
$$

The next theorem presents the proposed integrated guidance and control commands that ensure interception of a constant speed target.

Theorem 4. For a planar engagement between an endo-atmospheric interceptor and a constant speed target, whose engagement kinematics is governed by (1), with the dynamics of the interceptor described by (2) and the sliding manifold 
chosen as (11), the proposed integrated guidance and control commands

$$
\begin{aligned}
\delta_{c}^{c}= & \frac{L_{\delta_{c}} g_{2}^{\prime}\left(\alpha+\delta_{c}\right) \tau_{c} \tau_{t}^{2}}{L_{\delta_{c}}^{2} \tau_{t}^{2} g_{2}^{\prime 2}\left(\alpha+\delta_{c}\right)+L_{\delta_{t}}^{2} \tau_{c}^{2} g_{3}^{\prime 2}\left(\alpha+\delta_{t}\right) w^{2}}\left[-\frac{2 \tan \sigma}{V_{\mathrm{M}}} a_{\mathrm{M}}^{2}+\frac{2 V_{\mathrm{M}}}{r}\left(\dot{V}_{\theta}-\frac{V_{\theta}^{2} \tan \sigma}{r}\right)+\frac{4 V_{\theta}^{2} \tan \sigma-V_{r} V_{\theta}-r \dot{V}_{\theta}}{r V_{\theta}}\right. \\
& \left.-\Gamma q+\Gamma \frac{a_{\mathrm{M}}}{V_{\mathrm{M}}}+\frac{L_{\delta_{c}}}{\tau_{c}} g_{2}^{\prime}\left(\alpha+\delta_{c}\right) \delta_{c}+\frac{L_{\delta_{t}}}{\tau_{t}} g_{3}^{\prime}\left(\alpha+\delta_{t}\right) \delta_{t}-\frac{c V_{\theta} \sec ^{2} \sigma}{\eta}\left(V_{\theta}-\frac{r}{V_{\mathrm{M}}} a_{\mathrm{M}}\right)+\frac{V_{\mathrm{M}} \eta}{r V_{\theta} \sec ^{2} \sigma} \mu \operatorname{sign}(s)\right], \\
\delta_{t}^{c}= & \frac{L_{\delta_{t}} g_{3}^{\prime}\left(\alpha+\delta_{t}\right) \tau_{t} \tau_{c}^{2} w^{2}}{L_{\delta_{c}}^{2} \tau_{t}^{2} g_{2}^{\prime 2}\left(\alpha+\delta_{c}\right)+L_{\delta_{t}}^{2} \tau_{c}^{2} g_{3}^{\prime 2}\left(\alpha+\delta_{t}\right) w^{2}}\left[-\frac{2 \tan \sigma}{V_{\mathrm{M}}} a_{\mathrm{M}}^{2}+\frac{2 V_{\mathrm{M}}}{r}\left(\dot{V}_{\theta}-\frac{V_{\theta}^{2} \tan \sigma}{r}\right)+\frac{4 V_{\theta}^{2} \tan \sigma-V_{r} V_{\theta}-r \dot{V}_{\theta}}{r V_{\theta}}\right. \\
& \left.-\Gamma q+\Gamma \frac{a_{\mathrm{M}}}{V_{\mathrm{M}}}+\frac{L_{\delta_{c}}}{\tau_{c}} g_{2}^{\prime}\left(\alpha+\delta_{c}\right) \delta_{c}+\frac{L_{\delta_{t}}}{\tau_{t}} g_{3}^{\prime}\left(\alpha+\delta_{t}\right) \delta_{t}-\frac{c V_{\theta} \sec ^{2} \sigma}{\eta}\left(V_{\theta}-\frac{r}{V_{\mathrm{M}}} a_{\mathrm{M}}\right)+\frac{V_{\mathrm{M}} \eta}{r V_{\theta} \sec ^{2} \sigma} \mu \operatorname{sign}(s)\right]
\end{aligned}
$$

minimize the cost function, (17), subject to the constraint $\mathcal{B}_{c} \delta_{c}^{c}+\mathcal{B}_{t} \delta_{t}^{c}=-\mathcal{F}-\mu \operatorname{sign}(s)$, where $\mathcal{F}, \mathcal{B}_{c}$ and $\mathcal{B}_{t}$ are given by (25)-(26). Further, this guarantees that the interceptor converges to the pure deviated course within a finite-time, $t_{s}$, and results in an interception at a desired time, $T_{f}$.

Proof. We proceed in a similar fashion as in the proof of Theorem 2. Using the expressions of $\mathcal{F}, \mathcal{B}_{c}$ and $\mathcal{B}_{t}$, as in (25)-(26), minimization of the cost, (17), with $\mathcal{B}_{c} \delta_{c}^{c}+\mathcal{B}_{t} \delta_{t}^{c}=-\mathcal{F}-\mu \operatorname{sign}(s)=\Upsilon$ as a constraint, results in the guidance commands as the ones given in (21). On substituting the values of $\Upsilon, \mathcal{B}_{c}$ and $\mathcal{B}_{t}$ in (21), one may obtain the expressions of the canard and the tail surface deflections, given in (27) and (28), respectively. This concludes the proof.

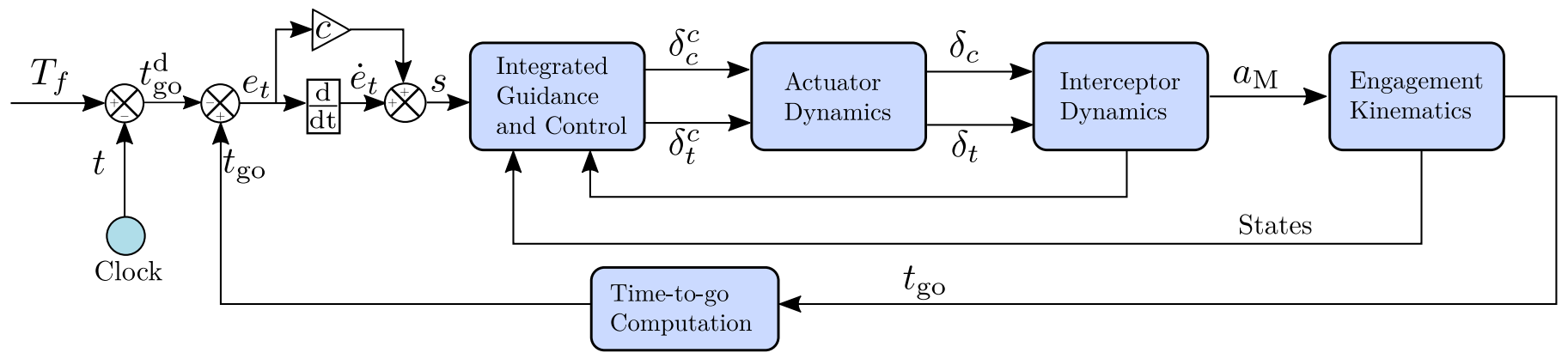

Figure 3: A schematic diagram of the proposed IGC design.

Interceptors are safety-critical systems in a realistic scenario, that is, even a minor failure in the system can lead to a catastrophe, resulting in mission failure. To avoid such situations, redundant actuators are often used which come into play after the main actuators have developed a fault. However, employing redundant actuators may require more space, better design efficiency, increased fuel and cost. A more in-depth consideration of such cases are currently beyond the scope of the current work. Nonetheless, we briefly discuss the case when a single actuator develops a fault at a certain time instant, $t_{1}<T_{f}$, leaving the sole onus of time-constrained interception on the other actuator. In this paper, we say that an actuator has developed a fault if it stops responding to the commanded value after $t_{1}$. For brevity, we shall consider that the tail develops some fault, while the canard remains operational throughout the engagement. In other words, the tail is unable to generate necessary deflection and remains stuck with its value at $t_{1}$ for all future time, that is, $\delta_{t}\left(t \geq t_{1}\right)=\delta_{t}\left(t_{1}\right)$ and $\delta_{t}^{c}\left(t \geq t_{1}\right)=0$. The canard, however, compensates for the tail using suitable deflections to generate the necessary lateral acceleration. Thus, the integrated guidance and control commands are modified, using 
Theorems 2 and 3, to accommodate such situations. For a stationary target interception, the command (18) becomes

$$
\begin{aligned}
\delta_{c}^{c}= & \frac{-\tau_{c}}{L_{\delta_{c}} g_{2}^{\prime}\left(\alpha+\delta_{c}\right)}\left[\frac{2 a_{\mathrm{M}}^{2}}{V_{\mathrm{M}} \tan 2 \sigma}+\frac{V_{\mathrm{M}}\left(K+2-5 \sin ^{2} \sigma\right)}{2 r \cos \sigma} a_{\mathrm{M}}+\frac{V_{\mathrm{M}}^{3} \tan \sigma}{2 r^{2}}\left(K+2-3 \sin ^{2} \sigma\right)+\Gamma q-\Gamma \frac{a_{\mathrm{M}}}{V_{\mathrm{M}}}\right. \\
& \left.-\frac{L_{\delta_{c}}}{\tau_{c}} g_{2}^{\prime}\left(\alpha+\delta_{c}\right) \delta_{c}-\frac{L_{\delta_{t}}}{\tau_{t}} g_{3}^{\prime}\left(\alpha+\delta_{t}\right) \delta_{t}^{\star}-\frac{c K V_{\mathrm{M}}^{2} \tan \sigma / 2}{2 r \cos \sigma}+\frac{c V_{\mathrm{M}}^{2} \sin \sigma}{2 r}+c a_{\mathrm{M}}+\frac{K V_{\mathrm{M}}^{2}}{r \sin 2 \sigma} \mu \operatorname{sign}(s)\right]
\end{aligned}
$$

and for interception of a constant speed target, the command (27) gets modified to

$$
\begin{aligned}
\delta_{c}^{c}= & \frac{\tau_{c}}{L_{\delta_{c}} g_{2}^{\prime}\left(\alpha+\delta_{c}\right)}\left[-\frac{2 \tan \sigma}{V_{\mathrm{M}}} a_{\mathrm{M}}^{2}+\frac{2 V_{\mathrm{M}}}{r}\left(\dot{V}_{\theta}-\frac{V_{\theta}^{2} \tan \sigma}{r}\right)+\frac{4 V_{\theta}^{2} \tan \sigma-V_{r} V_{\theta}-r \dot{V}_{\theta}}{r V_{\theta}}-\Gamma q+\Gamma \frac{a_{\mathrm{M}}}{V_{\mathrm{M}}}\right. \\
& \left.+\frac{L_{\delta_{c}}}{\tau_{c}} g_{2}^{\prime}\left(\alpha+\delta_{c}\right) \delta_{c}+\frac{L_{\delta_{t}}}{\tau_{t}} g_{3}^{\prime}\left(\alpha+\delta_{t}\right) \delta_{t}^{\star}-\frac{c V_{\theta} \sec ^{2} \sigma}{\eta}\left(V_{\theta}-\frac{r}{V_{\mathrm{M}}} a_{\mathrm{M}}\right)+\frac{V_{\mathrm{M}} \eta}{r V_{\theta} \sec ^{2} \sigma} \mu \operatorname{sign}(s)\right],
\end{aligned}
$$

where $\delta_{t}^{\star}$ denotes the value of $\delta_{t} \forall t>t_{1}$. Note that the canard failure can be dealt with in a similar fashion.

A schematic of the proposed integrated guidance and control design is illustrated in Fig. 3. The desired impact time, $T_{f}$, is compared with the current time to generate the desired time-to-go, which is again compared with the time-to-go at every instant to generate the impact time error. The time-to-go at every moment is obtained from the engagement kinematics. The kinematic information along with the sliding manifold, designed using the impact time error and its rate, and the states of interceptor are utilized by the IGC system to generate the commanded control surface deflections, that is, $\delta_{c}^{c}$ and $\delta_{t}^{c}$. These commanded signals are fed to the actuators, which generate the effective canard and tail surface deflections, $\delta_{c}$ and $\delta_{t}$. These further modify, as necessary, the angle of attack of the interceptor, and thus, achieve desired lift and lateral acceleration. Once the sliding mode is enforced, the interceptor maintains a course requisite for time-constrained interception, eventually intercepting the target at a desired time, $T_{f}$.

\section{Simulation Studies}

In this section, the theoretical claims in the preceding sections are validated using simulations for various engagement scenarios. We assume that the interceptor's aerodynamic surfaces, the canard and the tail, may exhibit a maximum bounded deflection of $40^{\circ}$ each. The nonlinear functions $g_{i}(\cdot), i=1,2, \ldots, 6$ are chosen as sigmoid functions, [12], with suitable boundary layers. The key parameters same across all the cases are presented in Table 1 , while the values of aerodynamic coefficients are chosen to be the same as those in [25].

In Fig. 4, the origin is assumed to be located at the interceptor's launch position while the stationary target is located at an initial LOS of $\theta(0)=0^{\circ}$. The interceptor is launched with a flight path angle of $\gamma_{\mathrm{M}}=10^{\circ}$. For an impact time of $30 \mathrm{~s}$, Fig. 4(a) depicts the trajectory of the interceptor in physical space, with a black circle marker representing the launch location and a red square marker representing the stationary target. It can be observed that the interceptor makes a detour initially until the time-to-go error is nullified, after which it attains the requisite course enabling an interception at the desired time. Fig. 4(b) shows the behavior of time-to-go estimate with the proposed scheme. In the given geometry, the initial time-to-go estimate is $25.08 \mathrm{~s}$, which is lower than the desired one. As soon as sliding mode is enforced, the time-to-go error converges to its desired value within 10 s. Since the lead angle is initially 
Table 1: Simulation parameters.

\begin{tabular}{ccc|ccc}
\hline Parameter & Values & Unit & Parameter & Values & Unit \\
\hline$V_{\mathrm{M}}$ & 400 & $\mathrm{~m} / \mathrm{s}$ & $r(0)$ & 10 & $\mathrm{~km}$ \\
$c$ & 1 & - & $K$ & 10 & - \\
$L_{\alpha}^{\beta}$ & 1190 & $\mathrm{~m} / \mathrm{s}^{2}$ & $M_{\alpha}^{\beta}$ & -100 & $\mathrm{~m} / \mathrm{s}^{2}$ \\
$L_{\delta_{c}}$ & 40 & $\mathrm{~m} / \mathrm{s}^{2}$ & $L_{\delta_{t}}$ & 40 & $\mathrm{~m} / \mathrm{s}^{2}$ \\
$M_{\delta_{c}}$ & 80 & $\mathrm{~s}^{-2}$ & $M_{\delta_{t}}$ & -80 & $\mathrm{~s}^{-2}$ \\
$M_{q}$ & -5 & $\mathrm{~s}^{-1}$ & $\alpha$ & 0 & $\mathrm{deg}\left(^{\circ}\right)$ \\
$\tau_{c}$ & 0.5 & $\mathrm{~s}$ & $\tau_{t}$ & 0.01 & $\mathrm{~s}$ \\
$\mu$ & 6 & - & $w$ & 1 & - \\
\hline
\end{tabular}

non-zero, and the desired time is greater than the initial time-to-go estimate, the lead angle increases in the reaching phase to $76.8^{\circ}$, and then decreases monotonically to zero in the sliding phase. This is shown in Fig. 4(c), along with the lateral acceleration profile, which is initially high, around $10 \mathrm{~g}$, to impose the proper interceptor's heading, but starts to tend to zero in the sliding phase. Similar phenomenon is exhibited by the aerodynamic surfaces, as portrayed in Fig. 4(d), demanding a high value at the beginning and a gradual reduction in demand with the onset of sliding phase. With initially high surface deflections, the angle of attack, and the pitch rate are also observed to be higher at the beginning, as shown in Figs. 4(e)-4(f). It should be noticed that once sliding mode occurs, interceptor's look angle, lateral acceleration, aerodynamic surface deflections, angle of attack, and the pitch rate, all tend toward zero with a zero terminal magnitude, which also helps in maximizing the speed of impact at the time of target interception.

With initial flight path angle set at $0^{\circ}$, the performance of proposed design is demonstrated in Fig. 5 for target interception at various impact times, starting 40,50 and $60 \mathrm{~s}$, whereas the initial time-to-go is $25 \mathrm{~s}$. It can be inferred from the behavior of interceptor's trajectories (in relative geometry) that until the time-to-go error converges, the interceptor goes slightly farther from the target to attain the desired collision geometry. As soon as the error converges, the interceptor takes a path that will lead to a time-constrained interception. In doing so, higher lateral acceleration is demanded in the beginning, but reduces thereafter.

The efficacy of proposed design is also demonstrated for various engagement geometries, by varying the interceptor's heading and the LOS angle, as illustrated in Fig. 6. Due to these variations, the initial time-to-go values are different in each of the three cases, and are found to be $25.62 \mathrm{~s}, 26.25 \mathrm{~s}$, and $25.45 \mathrm{~s}$. For $T_{f}=35 \mathrm{~s}$, an accurate tracking is observed and the error converges within $15 \mathrm{~s}$ for each of these cases. Similar conclusions can be drawn as in the previous cases, attesting the performance of the proposed IGC design. This also affirms that the proposed design, developed using nonlinear relations, remains valid for engagements with arbitrary initial geometry.

Next, we demonstrate the efficacy of the proposed IGC scheme to intercept a constant speed target, moving with $V_{\mathrm{T}}=300 \mathrm{~m} / \mathrm{s}$, at a time chosen a priori. Under the action of the proposed IGC commands, (27)-(28), Fig. 7 shows a time-constrained interception of a constant speed target moving with a heading of $\gamma_{\mathrm{T}}=120^{\circ}$. The LOS for the interceptor-target engagement in Fig. 7 is $0^{\circ}$, and the interceptor is launched with a flight path angle of $10^{\circ}$. In Fig. $7(\mathrm{a})$, a black circle marker represents the initial position of the endo-atmospheric interceptor, while magenta circle markers appear on the plot of the target every $10 \mathrm{~s}$ of engagement. With a desired time of interception set at $40 \mathrm{~s}$, it can 


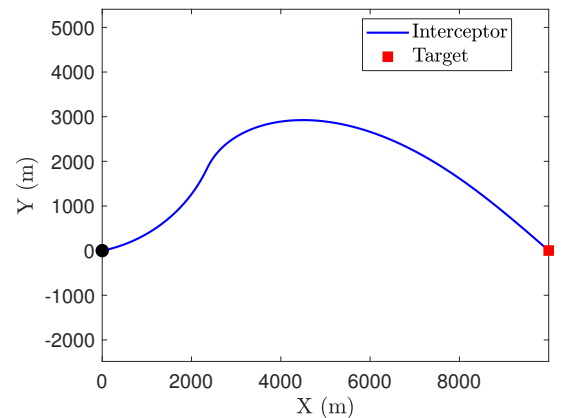

(a)

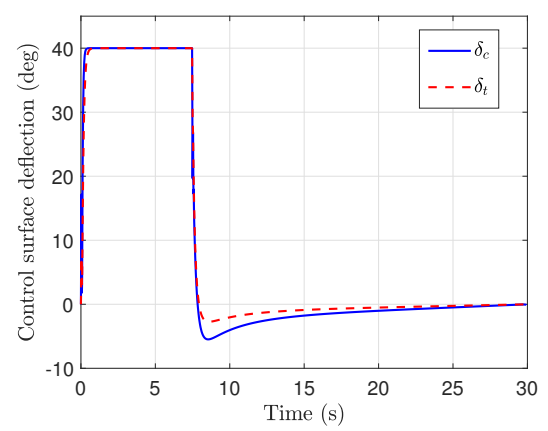

(d)

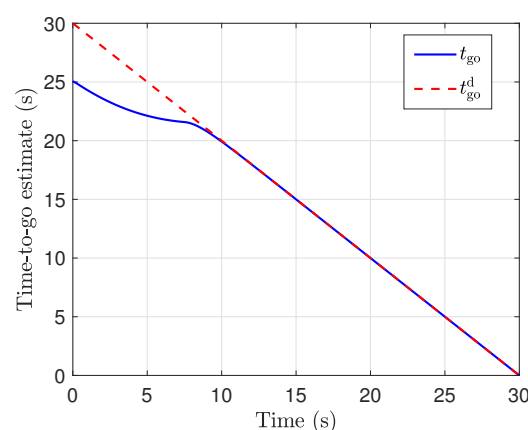

(b)

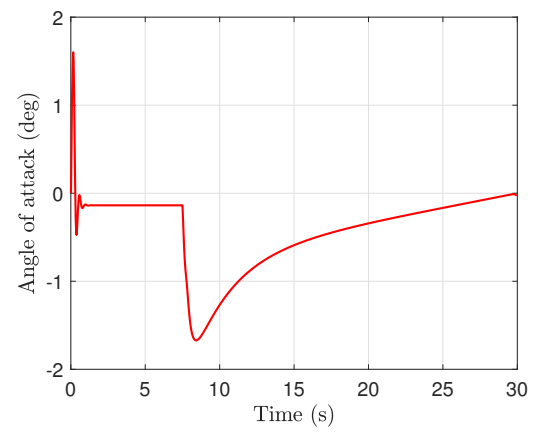

(e)
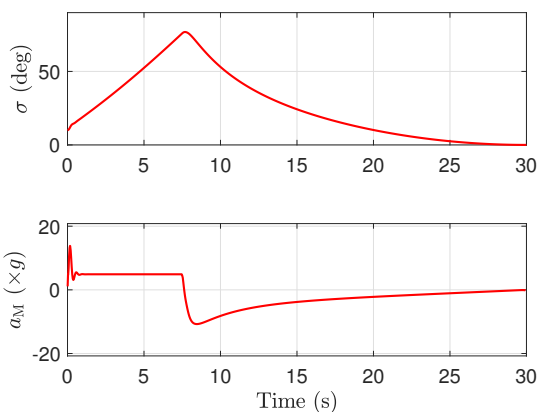

(c)

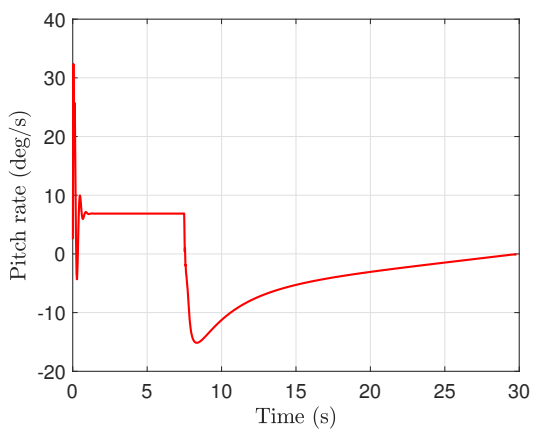

(f)

Figure 4: Interception of a stationary target at an impact time of $30 \mathrm{~s}$. (a) Trajectory. (b) Time-to-go estimate. (c) Lead angle and lateral acceleration. (d) Control surface deflections. (e) Angle of attack. (f) Pitch rate.

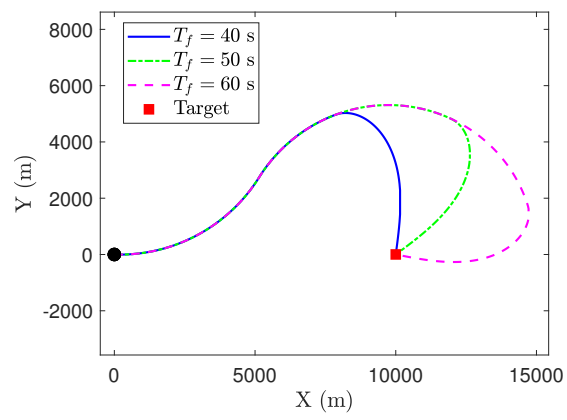

(a)

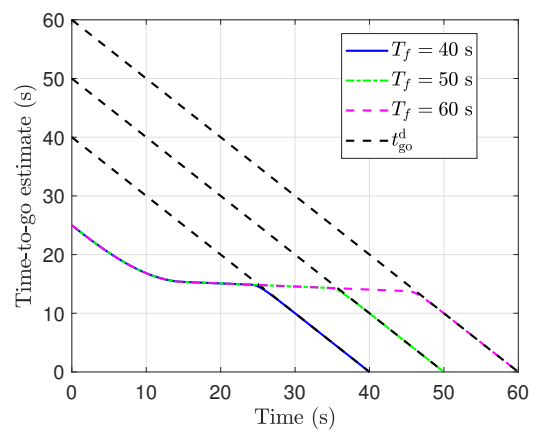

(b)
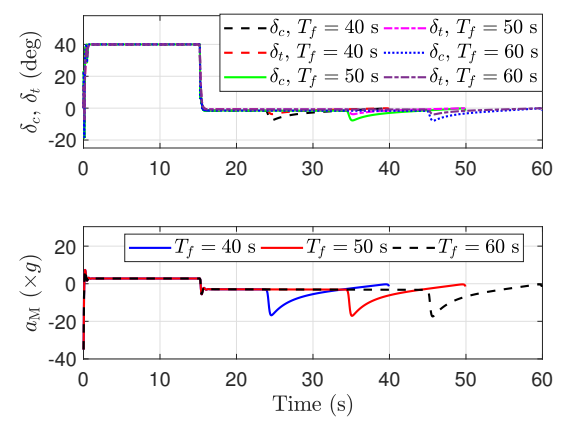

(c)

Figure 5: Interception of a stationary target for various impact time. (a) Trajectory. (b) Time-to-go estimate. (c) Control surface deflections and lateral acceleration.

be observed from Fig. 7(a) that a successful time-constrained interception occurs. The initial time-to-go, as seen from Fig. 7(b), is 30.05 s. As soon as sliding mode is enforced, the time-to-go tracks the desired profile in 5.45 s. During this time, the deviation angle (the look angle), $\sigma$, gets fixed, facilitating the time-constrained interception (see Fig. 7(c)). The lateral acceleration (Fig. 7(c)), the control surface deflections (Fig. 7(d)), the angle of attack (Fig. 7(e)) and the pitch rate (Fig. 7(f)) exhibit similar behavior after the error in impact time has converged to zero. Compared to the case of stationary target in Fig. 4, the control effort is lesser in the case of a constant speed target, shown in Fig. 7. This may be attributed to the exact nature of the time-to-go expression, (22), in the case of constant speed targets, as 


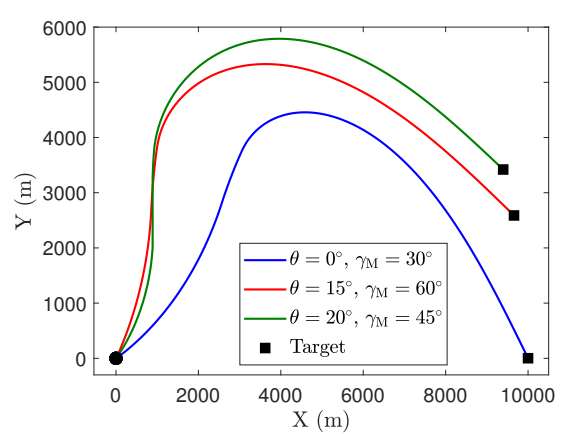

(a)

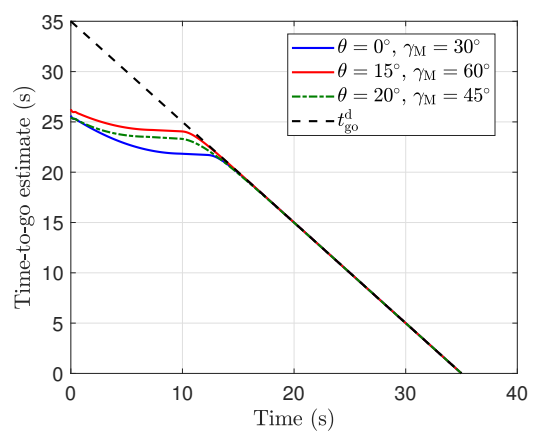

(b)
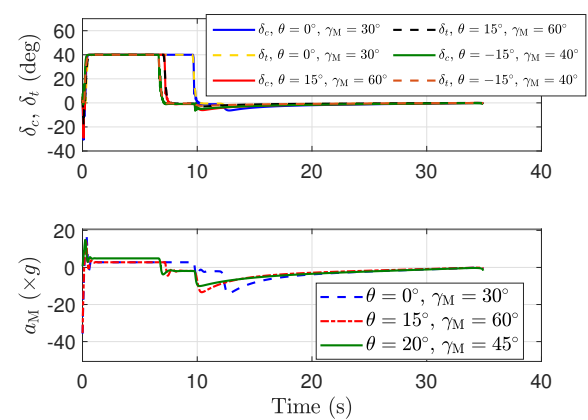

(c)

Figure 6: Interception of a stationary target under various geometries at an impact time of 35 s. (a) Trajectory. (b) Time-to-go estimate. (c) Control surface deflections and lateral acceleration.

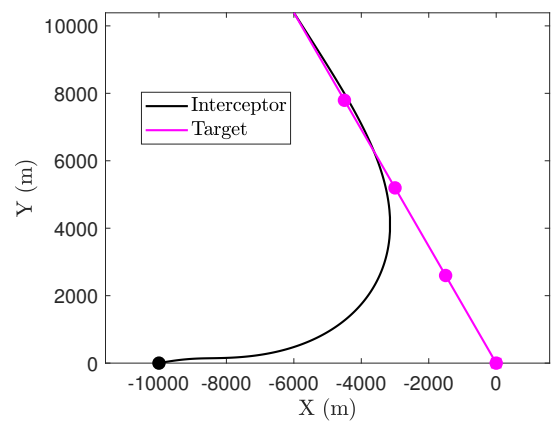

(a)

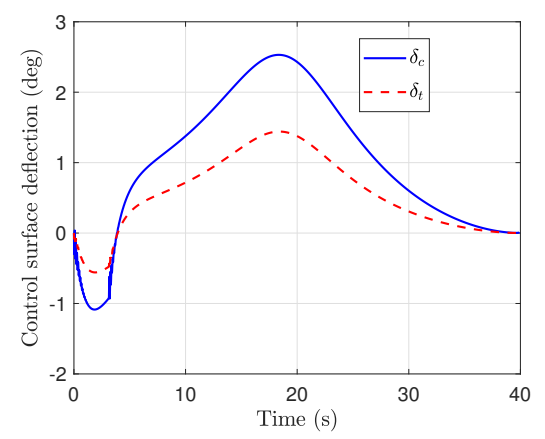

(d)

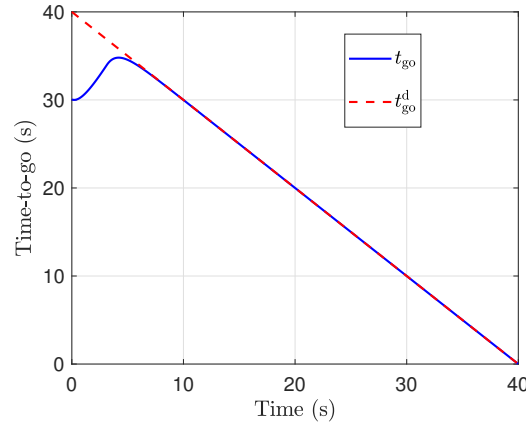

(b)

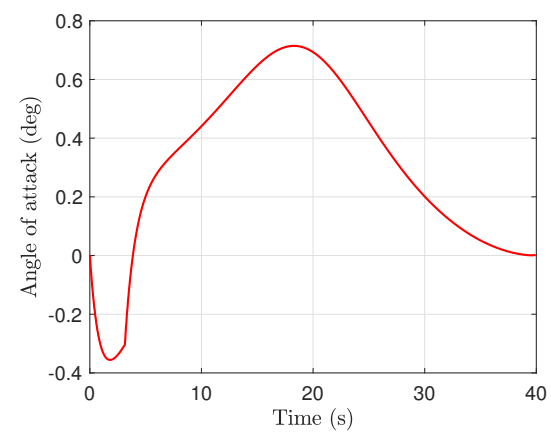

(e)
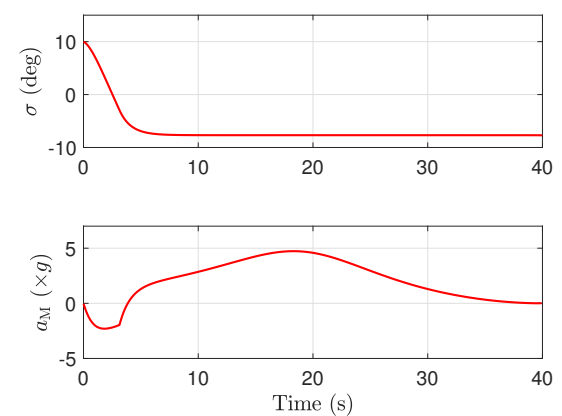

(c)

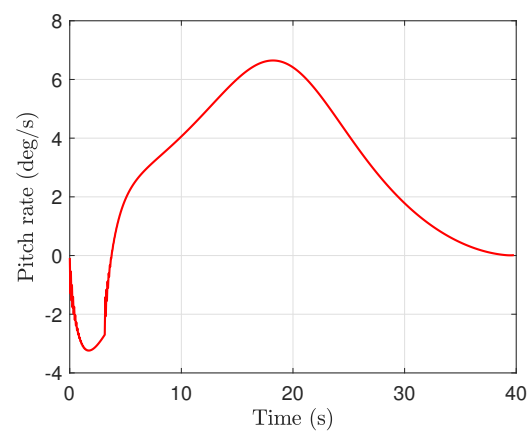

(f)

Figure 7: Interception of a constant speed target at an impact time of $40 \mathrm{~s}$. (a) Trajectory. (b) Time-to-go. (c) Deviation angle and lateral acceleration. (d) Control surface deflections. (e) Angle of attack. (f) Pitch rate.

opposed to the estimate, (7), in the case of stationary ones. It is also worth noting that the terminal demand of lateral acceleration and the aerodynamic surface deflections have zero magnitude. This is a desirable feature in any guidance design. This essentially means that once the interceptor has aligned itself on a course desirable for time-constrained interception, only a little effort is needed to maintain the course.

Fig. 8(a) depicts interception of a constant speed target at various impact time values. The flight path angles of the target and interceptor are $80^{\circ}$ and $180^{\circ}$, respectively, while the initial LOS angle is $-170^{\circ}$. Fig. 8(a) depicts various trajectories corresponding to each impact time. For a larger impact time, the interceptor takes a higher curvature path 


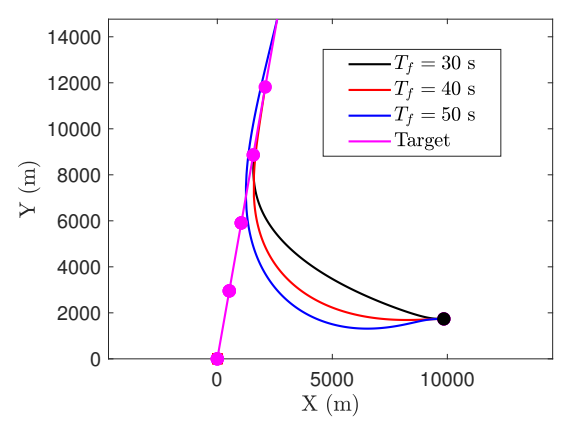

(a)

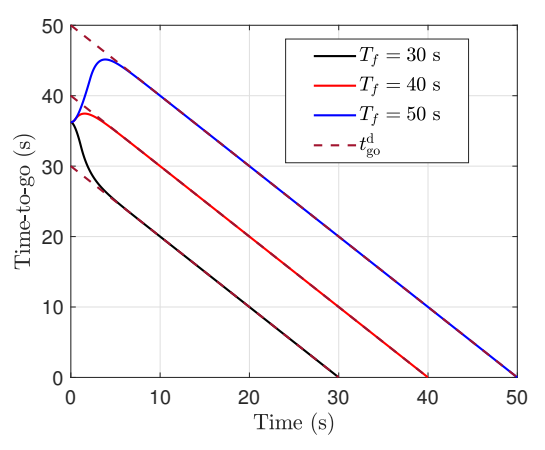

(b)
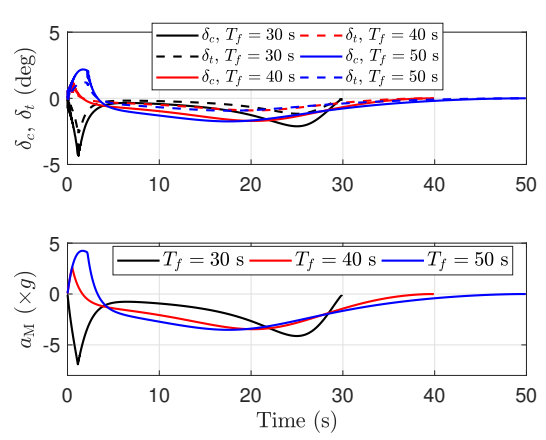

(c)

Figure 8: Interception of a constant speed target for various impact time. (a) Trajectory. (b) Time-to-go. (c) Control surface deflections and lateral acceleration.

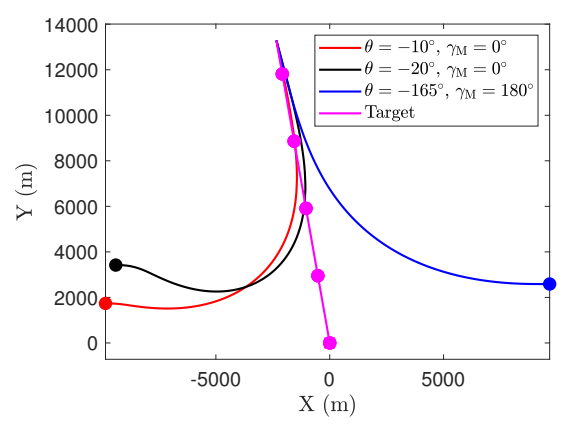

(a)

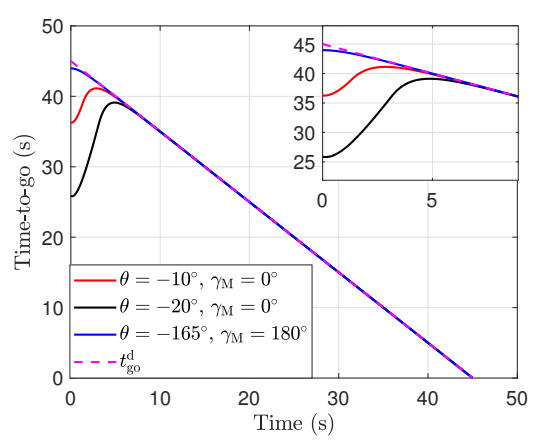

(b)
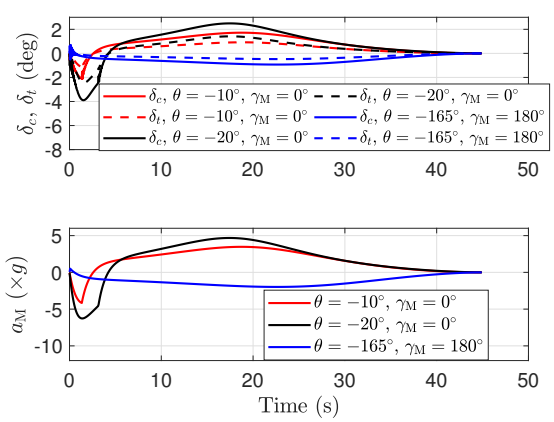

(c)

Figure 9: Interception of a constant speed target under various geometries at an impact time of 45 s. (a) Trajectory. (b) Time-to-go estimate. (c) Control surface deflections and lateral acceleration.

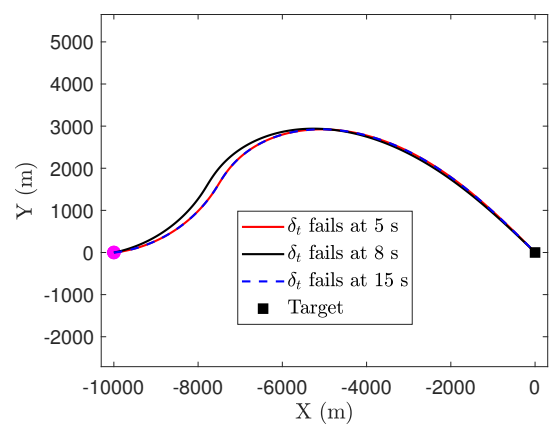

(a)

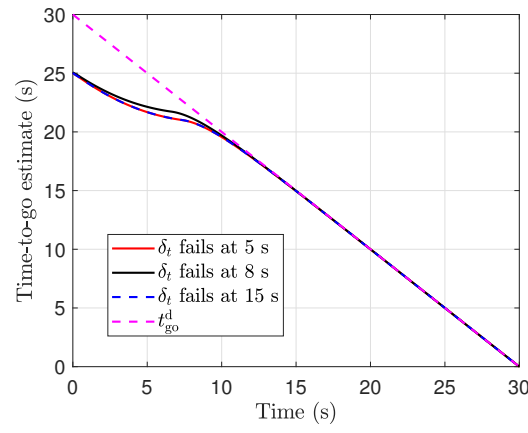

(b)
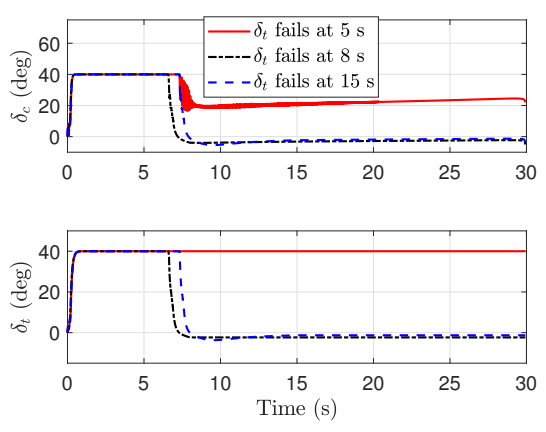

(c)

Figure 10: Interception of a stationary target with impaired actuator. (a) Trajectory. (b) Time-to-go estimate. (c) Control surface deflections.

initially until the impact time error is nullified. As soon as the time-to-go converges to the desired value, the interceptor converges to the pure deviated course with a fixed deviation angle. Fig. 8(b) portrays the time-to-go profiles for each impact time. It is remarkable that the proposed design not only enables a time-constrained interception at values higher than the initial time-to-go, but even lower than the initial time-to-go. This, clearly, vindicates the proposed design in satisfying a wide range of impact time. The behavior of the canard and the tail surfaces, along with that of the lateral acceleration, are depicted in Fig. 8(c), which show a tendency to reduce to zero after the convergence of time-to-go 


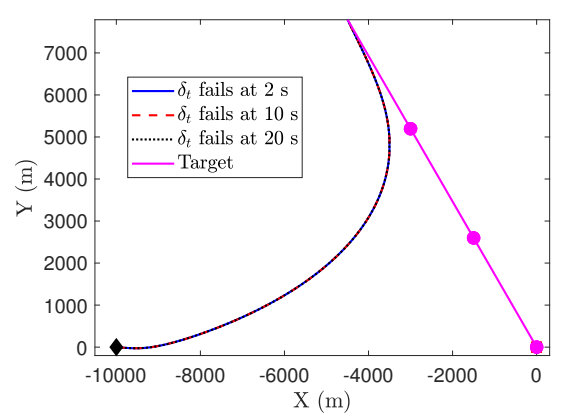

(a)

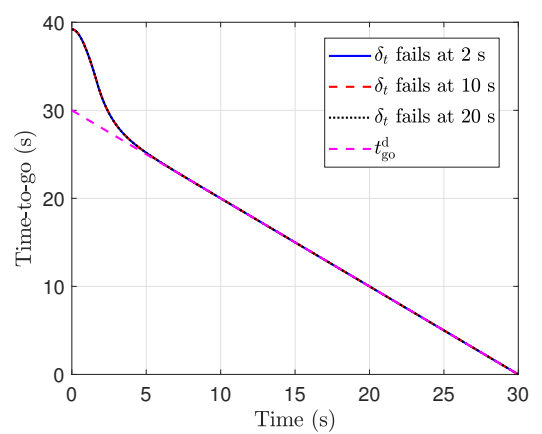

(b)
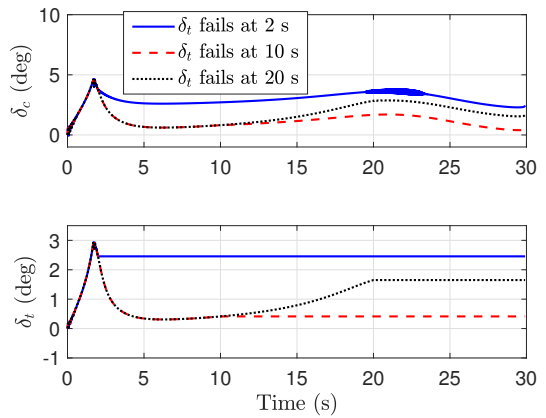

(c)

Figure 11: Interception of a constant speed target with impaired actuator. (a) Trajectory. (b) Time-to-go. (c) Control surface deflections.

error.

Similar to Fig. 6, the interception of the constant speed target from various engagement geometries, is illustrated in Fig. 9. It can be seen that the proposed IGC design is applicable over arbitrary initial engagement geometry. The initial time-to-go in this case are $36.27 \mathrm{~s}, 25.87 \mathrm{~s}$ and $43.98 \mathrm{~s}$, which converge to a desired value of $45 \mathrm{~s}$ with the enforcement of sliding mode. The behavior of aerodynamic surface deflections and the lateral acceleration follow a similar trend as in the previous cases.

Fig. 10 depicts the time-constrained interception of a stationary target with impaired tail for $T_{f}=30 \mathrm{~s}$. The initial conditions for the results shown in Fig. 10 are same as the ones chosen for Fig. 4. Three different fault time instants, $t_{1}$, are considered to demonstrate the effectiveness of the proposed design when the tail actuator fails. The time instant, $t_{1}=5 \mathrm{~s}$, denotes the time before the convergence of the time-to-go to the desired value, while $t_{1}=8 \mathrm{~s}$ is the instant when the time-to-go is just about to converge. Finally, $t_{1}=15 \mathrm{~s}$ is the time instant when the time-to-go has converged and the interceptor is on the collision geometry. Figs. 10(a) and 10(b) depict that the trajectories and the time-to-go for each case do not differ much. However, it is evident from Fig. 10(c) that the canard deflection is more when the tail fails before the time-to-go has converged. It is also worth noting that the terminal magnitude of canard is non-zero in such case. If the tail fails closer to the convergence of time-to-go or later in the engagement, then the behavior of $\delta_{c}$ is similar to the one in Fig. 4(d), with a zero terminal magnitude. In each case, $\delta_{t}$ does not change after $t_{1}$, as seen from Fig. 10(c).

For the case of tail failure, Fig. 11 portrays the interception of a constant speed target at $T_{f}=30$, with the same initial conditions as those used for Fig. 7, except $\gamma_{\mathrm{M}}$, which is $-5^{\circ}$ in this case. Similar to the case of tail failure in the stationary target interception, we show the effectiveness of the proposed commands for faults occurring before time-to-go convergence as well as after the time-to-go has tracked its desired value. The time-to-go, in this case, converges to its desired value within $5 \mathrm{~s}$. Noticeably, the behavior of the trajectories and time-to-go (see Figs. 11(a)-11(b)) are similar irrespective of the time, $t_{1}$, at which a fault occurs. The canard, however, tries to compensate for the tail contribution to ensure a time-constrained interception, as shown in Fig. 11(c). From the results shown in Figs. 10-11, it can be inferred that the proposed integrated guidance and control design exhibits some degree of robustness or a tolerance to incidents such as actuator failure. 


\section{Concluding remarks}

This work presented the idea of integrated guidance control design, using sliding mode control, to intercept a nonmaneuvering target at a desired impact time. To the best of the author's knowledge, such a scheme with impact time constraints did not exist in the literature earlier. A weighted allocation scheme provided additional flexibility in apportioning the available effort in both canard and tail configurations to generate the suitable lateral acceleration. Moreover, a general framework for choosing surface deflection was also provided, which opens up several possibilities in selection of these commanded control surface deflections. With some astute modifications, the additional control effort, either canard or tail, might be used to meet additional objectives. The complete nonlinear framework allowed the proposed scheme to remain applicable for a wide variety of engagement geometries, including those that induce significantly large heading errors. This also helped the proposed strategies to satisfy a wide range of impact time values. The actuator fault tolerance case was briefly discussed to show that the proposed design offers some degree of robustness to such scenarios.

Interception of maneuvering targets, along with additional terminal constraints, may be an interesting future extension of this work. The proposed integrated guidance and control design may also be utilized to design a cooperative salvo guidance in future.

\section{References}

[1] S. R. Kumar and D. Mukherjee, "Cooperative salvo guidance using finite-time consensus over directed cycles," IEEE Transactions on Aerospace and Electronic Systems, vol. 56, no. 2, pp. 1504-1514, 2020.

[2] E. J. Holder and V. B. Sylvester, "An analysis of modern versus classical homing guidance," IEEE Transactions on Aerospace and Electronic Systems, vol. 26, no. 4, pp. 599-606, 1990.

[3] D. Chwa and J. Y. Choi, "Adaptive nonlinear guidance law considering control loop dynamics," IEEE Transactions on Aerospace and Electronic Systems, vol. 39, no. 4, pp. 1134-1143, 2003.

[4] A. Zhurbal and M. Idan, "Effect of estimation on the performance of an integrated missile guidance and control system," IEEE Transactions on Aerospace and Electronic Systems, vol. 47, no. 4, pp. 2690-2708, 2011.

[5] X. Wang, C. P. Tan, and D. Zhou, "Observer-based PIGC for missiles with impact angle constraint," IEEE Transactions on Aerospace and Electronic Systems, vol. 55, no. 5, pp. 2226-2240, 2019.

[6] D. Chwa, "Robust nonlinear disturbance observer based adaptive guidance law against uncertainties in missile dynamics and target maneuver," IEEE Transactions on Aerospace and Electronic Systems, vol. 54, no. 4, pp. 1739-1749, 2018.

[7] I.-S. Jeon, J.-I. Lee, and M.-J. Tahk, "Impact-time-control guidance law for anti-ship missiles," IEEE Transactions on Control Systems Technology, vol. 14, no. 2, pp. 260-266, March 2006. 
[8] Min-Jea Tahk, Chang-Kyung Ryoo, and Hangju Cho, "Recursive time-to-go estimation for homing guidance missiles," IEEE Transactions on Aerospace and Electronic Systems, vol. 38, no. 1, pp. 13-24, 2002.

[9] I.-S. Jeon, J.-I. Lee, and M.-J. Tahk, "Homing guidance law for cooperative attack of multiple missiles," Journal of Guidance, Control, and Dynamics, vol. 33, no. 1, pp. 275-280, Jan 2010.

[10] M. Tahk, S. Shim, S. Hong, H. Choi, and C. Lee, "Impact time control based on time-to-go prediction for seaskimming antiship missiles," IEEE Transactions on Aerospace and Electronic Systems, vol. 54, no. 4, pp. 2043-2052, 2018.

[11] A. Saleem and A. Ratnoo, "Lyapunov-based guidance law for impact time control and simultaneous arrival," Journal of Guidance, Control, and Dynamics, vol. 39, no. 1, pp. 164-173, 2016.

[12] S. R. Kumar and D. Ghose, "Impact time guidance for large heading errors using sliding mode control," IEEE Transactions on Aerospace and Electronic Systems, vol. 51, no. 4, pp. 3123-3138, Oct 2015.

[13] D. Cho, H. J. Kim, and M.-J. Tahk, "Nonsingular sliding mode guidance for impact time control," Journal of Guidance, Control, and Dynamics, vol. 39, no. 1, pp. 61-68, May 2015.

[14] A. Sinha and S. R. Kumar, "Super-twisting control based cooperative salvo guidance using leader-follower approach," IEEE Transactions on Aerospace and Electronic Systems, pp. 1-10, 2020, early access, doi: 10.1109/TAES.2020.2974044.

[15] J. Zhou and J. Yang, "Guidance law design for impact time attack against moving targets," IEEE Transactions on Aerospace and Electronic Systems, vol. 54, no. 5, pp. 2580-2589, 2018.

[16] H. Kim, D. Cho, and H. J. Kim, "Sliding mode guidance law for impact time control without explicit time-to-go estimation," IEEE Transactions on Aerospace and Electronic Systems, vol. 55, no. 1, pp. 236-250, 2019.

[17] S. R. Kumar and D. Mukherjee, "Deviated pursuit based interception at a priori fixed time," Journal of Guidance, Control, and Dynamics, vol. 42, no. 9, pp. 2124-2131, 2019.

[18] N. Shneydor, Missile Guidance and Pursuit: Kinematics, dynamics and control. Cambridge, UK: Woodhead Publishing Limited, 1998, pp. 47-74.

[19] T. Shima, M. Idan, and O. M. Golan, "Sliding-mode control for integrated missile autopilot guidance," Journal of Guidance, Control, and Dynamics, vol. 29, no. 2, pp. 250-260, 2006.

[20] J. Guo, Y. Xiong, and J. Zhou, "A new sliding mode control design for integrated missile guidance and control system," Aerospace Science and Technology, vol. 78, pp. 54 - 61, 2018.

[21] B. Panchal, N. Mate, and S. E. Talole, "Continuous-time predictive control-based integrated guidance and control," Journal of Guidance, Control, and Dynamics, vol. 40, no. 7, pp. 1579-1595, 2017.

[22] M. Xin, S. N. Balakrishnan, and E. J. Ohlmeyer, "Integrated guidance and control of missiles with $\theta-D$ method," IEEE Transactions on Control Systems Technology, vol. 14, no. 6, pp. 981-992, 2006. 
[23] S. He, T. Song, and D. Lin, "Impact angle constrained integrated guidance and control for maneuvering target interception," Journal of Guidance, Control, and Dynamics, vol. 40, no. 10, pp. 2653-2661, 2017.

[24] J. Yun and C. Ryoo, "Integrated guidance and control law with impact angle constraint," in 2011 11th International Conference on Control, Automation and Systems, 2011, pp. 1239-1243.

[25] M. Idan, T. Shima, and O. M. Golan, "Integrated sliding mode autopilot-guidance for dual-control missiles," Journal of Guidance, Control, and Dynamics, vol. 30, no. 4, pp. 1081-1089, 2007.

[26] H. Yan and H. Ji, "Integrated guidance and control for dual-control missiles based on small-gain theorem," Automatica, vol. 48, no. 10, pp. 2686 - 2692, 2012.

[27] D. K. Giri and M. Sinha, "Finite-time continuous sliding mode magneto-coulombic satellite attitude control," IEEE Transactions on Aerospace and Electronic Systems, vol. 52, no. 5, pp. 2397-2412, 2016. 